\title{
Biochemical markers of bone tissue metabolism in broiler chickens
}

\author{
Marina Semenenko ${ }^{1 *}$, Elena Kuzminova $^{1}$, Ksenia Semenenko ${ }^{1}$, Artem Vlasenko ${ }^{1}$, and Inna \\ Zholobova $^{2}$ \\ ${ }^{1}$ Krasnodar Research Center for Animal Husbandry and Veterinary Medicine, Pervomaiskaya st. 4, \\ Krasnodar, 350055, Russian Federation \\ ${ }^{2}$ Kuban State Agrarian University named after I.T. Trubilin, Kalinina st. 13, Krasnodar, 350044, \\ Russian Federation
}

\begin{abstract}
The article presents the results of studies to identify possible intravital markers of bone metabolism in fast-growing meat crosses. As a result of the research it was determined that the introduction of mineral components containing calcium, phosphorus, magnesium and silicon into the feed of broiler chickens on the background of the diet balanced in biologically active substances helps to activate the processes of biological synthesis in bone tissue, normalize its mineral component with a high degree of silicon assimilation and also increases the stability of the bones of the lower limbs of broiler chickens. It has been proved that in order to monitor the prevention and treatment of dyschondroplasia in meat poultry, it is necessary to use in vivo biochemical markers of bone tissue metabolism, which allow carrying out early and accurate diagnosis of bone diseases in poultry.
\end{abstract}

\section{Introduction}

Today, the dynamically developing branch of animal husbandry is industrial poultry farming, which provides the use of highly productive breeds and crosses of meet poultry and can make a significant contribution to the development of the country's agro-industrial complex $[1,2]$. However, as it turned out, the intensive increase in body weight of broiler chickens from the first days of life almost always outstrips the growth and development of bone tissue, the formation of which is associated with the features of mineralization in osteogenesis, which leads to the negative metabolic changes in the body of poultry. That is why degenerative bone tissue disorders develop in highly productive broilers, which is manifested by dyschondroplasia syndrome (weakness of the lower limbs) [3, 4]. Tibial dyschondroplasia (TD) is a metabolic disease of young poultry that affects the growth of bones and cartilage. It is common in broilers (chickens raised for meat) and other poultry that have been raised for rapid growth. The tibial cartilage does not mature enough to ossify (turn into bone). This makes the growth plate prone to fractures, infection and the development of bone deformities. Due to the large muscle mass of poultry, the center of gravity of the body shifts, which leads to the vulnerability of the proximal femur, while the weakness of the bone tissue, unable to

* Corresponding author: sever291@,mail.ru 
withstand the increased load, causes the development of clinical symptoms such as lameness, uncertain gait, pain and discomfort [5,6].

All processes occurring in bone tissue, both physiological and pathological, can be monitored and diagnosed using biochemical markers of bone tissue. These markers allow identifying a decrease in bone mass and assessing the risks of bone fractures; providing informative data on the average rate of skeletal remodeling as a whole; they are also important parameters for the development and evaluation of the effectiveness of the skeletal system diseases treatment; and they are necessary for carrying out effective measures for the prevention of osteopathologies in poultry meat farming [7-8].

These markers should include, first of all, such macronutrients as calcium, manganese and phosphorus. It is known that calcium in adequate amounts guarantees bone strength, and manganese regulates the growth and formation of the organic matrix, contributing to the deposition of calcium in bones.

An additional factor that determines the degree of calcium absorption from mineral sources is vitamin $\mathrm{D}_{3}$, which increases the retention of $\mathrm{Ca} 2+$ ions by bone tissue, the absorption of sulfur by chondrocytes during the formation of cartilage tissue and by osteocytes during the synthesis of osseomucoids and ossein. Vitamin D is an inducer of calcium-binding protein synthesis, which transports $\mathrm{Ca} 2+$ ions across epithelial cell membranes. Vitamin D-induced calcium-binding protein contains not only in the intestinal mucosa, but also in bone tissue, pancreas and other organs. Vitamin D also stimulates the absorption of inorganic phosphorus in the duodenum of animals [9,10,11].

Adequate supply of the poultry body with calcium, phosphorus and vitamin D is an important condition for the integrity of the bone tissue, but does not compensate all its nutritional needs. At the same time, recent studies in medicine have shown that for maintaining the structure of the organic matrix of bone tissue, a necessary and essential element is silicon, which is an osteogenic micronutrient that is directly involved in the mechanisms of growth and repair of osteocytes. Its maximum concentration is found inside the active bone cells - the cytoplasm of osteoblasts (in mitochondria) involved in the formation of intercellular substance and localized in the area of active growth of young bones. The formation of bone tissue depends on the activity of osteoblasts, which synthesize organic matrix and modify the processes of calcification. At the stage of bone tissue formation, the concentration of silicon compounds is 25 times higher than in the adjacent areas, decreasing as the degree of bone matrix mineralization increases (at a young age with active growth and bone formation, silicon increases the calcification rate in 1.5 times, improving inclusion of calcium in bone tissue). Whereas its lack leads to the fact that calcium, displacing the synovial fluid from the joints, settles in them in the form of salts, being one of the causes of arthrosis [12-15].

Recent studies have shown that a biochemical marker such as alkaline phosphatase (ALP) can be used as a general indicator of skeletal development in vertebrates. In poultry, there are age-related variations in ALP activity, presumably due to the processes of bone formation. Foreign studies have shown that the activity of the bone alkaline phosphatase isoform (bone ALP) is closely related to the overall rate of skeleton mineralization in young poultry. Moreover, the alkaline phosphatase activity in bones predicted the rate of mineralization of the bones of the legs and wings. The alkaline phosphatase isoform in liver was only marginally associated with the overall rate of skeletal mineralization, while there was no any connection with the long bone mineralization. Researchers concluded that plasma alkaline phosphatase activity in bones is a reliable biomarker of skeletal mineralization in poultry [16].

Thus, the aim of this research was to study possible biochemical markers of bone tissue metabolism in fast-growing meat poultry crosses. 


\section{Materials and Methods}

The studies were carried out on poultry of the Arbor Acres cross, divided into two groups experimental and control $(\mathrm{n}=35)$. As a mineral source of calcium, phosphorus, manganese and silicon, bentonite of the Kantemirovskiy deposit of the Voronezh region was used, which belongs to a rare variety of the silicite group. The composition of bentonite is shown in Table 1.

Table 1. The concentration of macro- and microelements in the bentonite of the Kantemirovskiy deposit.

\begin{tabular}{|c|c|}
\hline Indicator & Concentration of elements, mg / kg \\
\hline $\mathrm{Ca}$ & 45.0 \\
\hline $\mathrm{P}$ & 3.6 \\
\hline $\mathrm{Mn}$ & 85.0 \\
\hline $\mathrm{Fe}$ & 5.5 \\
\hline $\mathrm{Si}$ & 37.2 \\
\hline $\mathrm{Cu}$ & 23.1 \\
\hline $\mathrm{Zn}$ & 68.6 \\
\hline $\mathrm{J}$ & 0.15 \\
\hline $\mathrm{Mg}$ & 363.0 \\
\hline $\mathrm{Co}$ & $4.0-4.5$ \\
\hline
\end{tabular}

In addition to the above mentioned minerals, bentonite contains a number of macro- and microelements, presented in the form of compounds easily assimilated by the body [12].

The poultry was kept in single-tier cages with a mesh floor, trough feeders, nipple and vacuum drinkers under 24-hour light conditions. The poultry were fed with a complete feed balanced in vitamin $\mathrm{D}_{3}$ according to the following scheme: from the moment of birth to 10 days of life - start feed; from 11 to 28 days - growth feed; from day 29 to slaughter - finish feed. Experimental group daily got bentonite at the rate of $2 \%$ to the mass of feed from 1 to 42 days of life in the complete feed (CF). The control group got only complete feed. At the end of the experiment, blood was taken from each broiler from the group for biochemical analysis.

Biochemical studies of blood serum were carried out on an automated Vitalab Selectra Junior analyzer with software version 1.0. (an open system for photometric tests, manufactured by Vital Scientific N. V. Netherlands) using reagents from ELITech Clinical Systems (France) and Analyticon biotechnologies AG (Germany). The level of silicon in blood serum was measured at the atomic adsorption spectrometer "Kvant-2" (Russia).

Determination of the greatest limiting load on the bones of the lower limbs was carried out using mechanical compression with an electrohydraulic test press "PI-5000 kN" with a force from $1 \mathrm{kN}(100 \mathrm{~kg})$ to $5000 \mathrm{kN}$ (500 tons).

Statistical data processing was carried out using the Statistica v. 6. The criterion of reliability was determined according to the Student's table.

\section{Results}

According to the results of the study, the safety of broiler chickens throughout the experiment in both groups was $100 \%$. However, the observation of the clinical state of the experimental and control poultry was indicative. In the experimental group all poultry was more active during the entire research period with good feathering speed and even feathering. Loss of feathers in limited areas was not observed, the feather was held firmly in the skin. The comb and wattles were elastic and shiny. In the control group in the last 2 
weeks of the experiment, poultry with low mobility, ruffled feathering and dimly colored comb and wattles were registered. Weakened broilers found it more difficult to approach the feeders, which affected their intensity of growth. Starting from the third week of feeding in this group, a steady dynamics of a decrease in body weight gains began to appear - by $4.3 \%$ at 28 th day and by $7.4 \%$ at 42 nd day of life ( $\mathrm{p} \leq 0.05)$. Whereas the average daily body weight gain in the experimental group for all studied growing periods exceeded the control analogs by $8.2 \%, 11.7 \%(\mathrm{p} \leq 0.01)$ and $7.8 \%(\mathrm{p} \leq 0.05)$, respectively.

The results of the biochemical study of blood serum determined that the introduction of bentonite into the feed diet had a pronounced effect on the biochemical homeostasis of the blood of poultry (Table 2).

Table 2. The results of biochemical analysis of the blood of broiler chickens ( $M \pm m ; n=5)$.

\begin{tabular}{|c|c|c|}
\hline \multirow{2}{*}{ Indicators } & \multicolumn{2}{|c|}{ Groups } \\
\hline & Experimental & Control \\
\hline Total protein, $\mathrm{g} / \mathrm{l}$ & $32.35 \pm 0.25$ & $28.97 \pm 1.12$ \\
\hline Urea, $\mathrm{mmol} / \mathrm{l}$ & $2.60 \pm 0.06$ & $2.67 \pm 0.03$ \\
\hline Creatinine, $\mu \mathrm{mol} / \mathrm{l}$ & $20.73 \pm 0.46$ & $27.80 \pm 4.95$ \\
\hline Glucose, $\mathrm{mmol} / \mathrm{l}$ & $12.17 \pm 0.48^{* *}$ & $9.00 \pm 1.08$ \\
\hline Cholesterol, mmol / 1 & $3.57 \pm 0.09 * * *$ & $3.03 \pm 0.12$ \\
\hline Triglycerides, mmol / 1 & $0.50 \pm 0.26$ & $0.95 \pm 0.48$ \\
\hline Total bilirubin, $\mu \mathrm{mol} / 1$ & $8.57 \pm 0.37$ & $7.53 \pm 0.38$ \\
\hline Direct bilirubin, $\mu \mathrm{mol} / 1$ & $0.67 \pm 0.17$ & $0.90 \pm 0.12$ \\
\hline $\mathrm{AST}, \mathrm{AU}$ & $278.67 \pm 38.76$ & $244.33 \pm 16.18$ \\
\hline $\mathrm{ALT}, \mathrm{AU}$ & $7.00 \pm 1.00$ & $6.67 \pm 0.88$ \\
\hline ALP, AU & $187.6 \pm 9.16^{*}$ & $164.1 \pm 15.2$ \\
\hline Chlorides, mmol / 1 & $110.97 \pm 1.31 *$ & $106.73 \pm 1.13$ \\
\hline Total calcium, mmol / l & $2.97 \pm 0.03$ & $2.90 \pm 0.10$ \\
\hline Manganese, $\mathrm{mmol} / \mathrm{l}$ & $0.32 \pm 0.07$ & $0.21 \pm 0.01$ \\
\hline Vitamin $\mathrm{D}_{3}, \mathrm{nmol} / 1$ & $71.8 \pm 6.54 *$ & $59.6 \pm 9.42$ \\
\hline Inorganic phosphorus, mmol / 1 & $2.20 \pm 0.00^{* *}$ & $2.63 \pm 0.13$ \\
\hline Silicon, $\mu \mathrm{g} / 1$ & $732.33 \pm 22.57 * * *$ & $282.30 \pm 48.95$ \\
\hline
\end{tabular}

The degree of reliability in relation to the control: *** $-\mathrm{p} \leq 0.001,{ }^{*}-\mathrm{p} \leq 0.01, *-\mathrm{p} \leq 0.05$

During the experiment the total protein level in the experimental group increased by $11.7 \%$, the glucose concentration increased by $35.2 \%(\mathrm{p} \leq 0.01)$, blood cholesterol increased by $17.8 \%(\mathrm{p} \leq 0.001)$.

An increase in glucose in the blood serum of an experimental poultry can be considered as a manifestation of one of the mechanisms of activation of the energy-forming function of liver cells, in which the deposited glycogen is spent to maintain a higher level of metabolic processes in the body, as well as to intensify the cellular metabolism in hepatocytes. In addition, a high metabolic supply with a source of readily available energy is a characteristic feature of fast-growing animals, which include modern cross-breeds of meat poultry. However, the energy metabolic demand of broiler chickens is so high that the carbohydrate complex does not fully provide the plastic processes with energy at the celluler and subcellular levels. Therefore, in the experimental group, lipids were also involved in carbohydrate metabolism, that was confirmed by lower values of triglycerides in the blood of chickens (in 1.9 times).

A lower concentration of creatinine (an intermediate metabolite and an indicator of catabolic processes in muscles and liver) in the blood serum of experimental chickens indicates a higher level of plastic processes. This was indirectly confirmed by the change in the concentration of aminotransferases in experimental chickens, and, in particular, AST, the level of which increased by $14.1 \%$ relative to the control analogs. Moreover, the increase in AST activity occurred within the framework of physiological values. 
The activity of alkaline phosphatase in the blood serum of the experimental poultry was more pronounced. Intergroup differences were $14.3 \%$. It should be noted that, in contrast to mammals, in broiler chickens the body contains more isozyme of bone, rather than hepatic ALP, and its increase is usually associated with the increase in osteoblastic activity. In our case, an increase in this enzyme in experimental chickens most likely indicates a higher remodeling of bones, primarily of the lower limbs.

In the mineral metabolism of chickens from the experimental group, an insignificant increase in the concentration of total calcium (by 2.4\%) with a simultaneous decrease in the level of inorganic phosphorus (by 16.3\%) was determined. Taking into account the close relationship of these macroelements in metabolic processes, their ratio is also important, which in the groups was 1.35 (experimental) and 1.1 (control). The intensive growth of the poultry leads to a constantly increasing consumption of calcium for the formation of bone tissue of the skeleton and the physiological functioning of other organs. In this case, in the body of the experimental poultry the calcium-phosphorus ratio remained within more optimal limits, while the increase in the quantitative content of phosphorus in control broiler chickens suggests that there is a violation of bone mineralization due to insufficient absorption of calcium (which was experimentally confirmed with assessment of the physical characteristics of the bones of the lower limbs of the experimental poultry).

The concentration of manganese in the blood of the experimental chickens exceeded the similar values of the control poultry in 1.52 times, the level of vitamin $\mathrm{D}_{3}-$ by $20.5 \%$ $(\mathrm{p} \leq 0.05)$.

The dynamics of silicon concentration in the blood serum of broiler chickens had significant fluctuations between the experimental and control poultry. The intergroup differences were 2.59 times higher in favor of the experimental chickens with a high degree of reliability of the obtained results $(\mathrm{p} \leq 0.001)$. The bentonites of the Kantemirovskiy deposit are distinguished by the fact that in their chemical composition they contain a high percentage of amorphous silica (up to $37.2 \%$ ), which is largely absorbed by the poultry body, providing a high concentration of this microelement in the blood.

The next stage of the research was the study of the morphometric parameters of the bones of the lower limbs of the experimental poultry, for which, on the 42nd day of the study, control slaughter of poultry was carried out from each group by the method of random sampling ( 6 individuals from the group -3 females and 3 males) with complete extraction of internal organs and anatomical cutting. Before testing, the femur and tibia bones were cleaned of muscle tissue and tendons and subjected to degreasing, after which they were cut at an angle of $90^{\circ}$ in order to separate the diaphysis in the form of cylinders $3 \mathrm{~cm}$ long to give a stable position when placed in the device. The bone marrow was removed from the bones. Further, the diaphysis of the femur and tibia bones of the control and experimental groups were compressed on an electrohydraulic press in the vertical direction (in the plane simulating the natural load of body weight on the bone apparatus of the poultry limbs). The prepared bone cylinders were subjected to a load of $1 \mathrm{kN}$ or more. Compression was carried out until the destruction of the bone cylinder. At the moment of the fault, the maximum ultimate load on the object was recorded. The research results are presented in Table 3.

Table 3. Dynamics of the maximum limiting load of the femur and tibia bones of broiler chickens with a double-column floor test press $(\mathrm{M} \pm \mathrm{m} ; \mathrm{n}=3)$.

\begin{tabular}{|c|c|c|}
\hline \multirow{2}{*}{ Indicator } & \multicolumn{2}{|c|}{ Group } \\
\cline { 2 - 3 } & Experimental & \multicolumn{1}{|c|}{ Control } \\
\hline \multirow{3}{*}{ Maximum ultimate load $(\mathrm{kN})$} & \multicolumn{2}{|c|}{ Femur } \\
\cline { 2 - 3 } & $1.82 \pm 0.15$ & \multicolumn{2}{|c|}{$1.38 \pm 0.26$} \\
\cline { 2 - 3 } & \multicolumn{2}{|c|}{$1.30 \pm 0.18$} \\
\cline { 2 - 3 }
\end{tabular}

The degree of reliability: $* * * \mathrm{p} \leq 0.001$ in relation to control. 
Analyzing the results of the table it can be noted that the resistance of the femur bones to mechanical pressure with the subsequent destruction of the bone structure in broiler chickens of the experimental group was $31.9 \%$ higher, and the resistance of the tibia bones was 1.68 times higher. At the same time, the destruction of bone tissue in the experimental poultry proceeded smoothly, without jumps, in direct proportion to the compression force, while in the chickens of the control group with a sufficiently low mechanical load, a sharp deformation of the bone tissue was observed, leading to its fracture and subsequent destruction.

\section{Conclusion}

The carried out studies prove that in order to monitor the prevention and treatment of bone system disorders in dyschondroplasia in meat poultry, it is necessary to use biochemical markers of bone tissue metabolism, such as calcium, phosphorus, silicon, vitamin D and bone isoenzyme of alkaline phosphatase, which will simultaneously characterize the formation and bone resorption for the diagnosis of pathological conditions of bone tissue, followed by timely correction of bone metabolism disorders. Some of the analyzed markers can be considered as standard and generally accepted in the practice of veterinary medicine, while others require further research with the expansion of the evidence base. However, in all cases, the advantage of biochemical research methods is non-invasiveness and early diagnostic component with a high degree of obtaining reliable information.

\section{References}

1. D. Kuppusamy, International Journal of Economic and Business Review, 3(8), 63-67 (2015)

2. J. Biesek, J. Kuźniacka, M. Banaszak, S. Kaczmarek, M. Adamski, A. Rutkowski, M. Hejdysz, Animals, 10(5), 846 (2020) doi: 10.3390 / ani10050846 Doi: 10.3390 / ani10050846

3. S. Shetty, N. Kapoor, J.D. Bondu, N. Thomas, T.V. Paul, Indian J Endocrinol Metab., 20(6), 846-852 (2016) doi:10.4103/2230-8210.192914

4. Y. Abe, M. Chiba, S. Yaklai, et al., Sci Rep, 9, 20106 (2019) https://doi.org/10.1038/s41598-019-56484-X

5. M. Lorentzon, J. Branco, M.L. Brandi et al., Adv Ther., 36, 2811-2824 (2019) https://doi.org/10.1007/s12325-019-01063

6. H. Paxton, P.G. Tickle, J.W. Rankin, J.R. Codd, J.R. Hutchinson, Peer J., 2, 473 (2014) https://doi.org/10.7717/peerj.473

7. C.M. Romero Barco et al., Utilidad en la práctica clínica. Reumatol Clin. 8, 149-52 (2012) doi: 10.1016 / j.reumae.2011.05.004

8. T. Vilaca, F. Gossiel, R. Eastell, Journal of Clinical Densitometry, 20(3), 346-352 (2017) https://doi.org/10.1016/j.jocd.2017.06.020

9. Y. Guo et al., Poultry science, 98 (10), 4433-40 (2019) https://doi.org/10.3382/ps/pez269

10. Y. Huang, E. Eapen, S. Steele, V. Grey, Clinical biochemistry, 44(10-11), 771-778 (2011) 
11. E.M. Lewiecki, Therapeutic advances in musculoskeletal disease, 6(2), 48-57 (2014) doi: 10.1177 / 1759720X13510479

12. L.F. Rodella et al., The journal of nutrition, health \& aging, 18.9, 820-826 (2014)

13. M-H. Kim et al., Biological trace element research, 158.2, 238-242 (2014)

14. M. Dong et al., Biological trace element research, 173(2), 306-315 (2016)

15. M.P. Semenenko, E.V Kuzminova, A.G. Koshchaev, Advances in Agricultural and Biological Sciences, 1 (2), 3-10 (2015)

16. T. P. Gade, M. W. Motley, B. J. Beattie, R. Bhakta, A. L. Boskey, J.A. Koutcher, P. Mayer-Kuckuk, PloS one, 6(7), e22608 (2011)

https://doi.org/10.1371/journal.pone.0022608 